\title{
Avaliação radiográfica de pacientes portadores de escoliose idiopática do adolescente submetidos à instrumentação híbrida posterior tipo Universal Spine System (USS I)
} Radiologic analysis of patients with adolescent idiopathic scoliosis submitted to Universal Spine System (USS I) hybrid instrumentation Evaluación radiográfica de pacientes portadores de escoliosis idiopática del adolescente sometidos a la instrumentación híbrida posterior tipo Universal Spine System (USS I)

\author{
Alexandre Roberto Aprile ${ }^{1}$ \\ Maria Fernanda Silber Caffaro 2 \\ Robert Meves ${ }^{3}$ \\ Elcio Landim ${ }^{4}$
}

\section{RESUMO}

Objetivo: analisar e comparar aos achados da literatura os desfechos da correção radiográfica no plano coronal em adolescentes portadores de escoliose idiopática torácica que foram submetidos ao tratamento cirúrgico com instrumental de terceira geração tipo Universal Spine System (USS I). Desenho do estudo: estudo transversal. Série de casos. Métodos: vinte adolescentes portadores de escoliose idiopática do adolescente foram submetidos à artrodese com instrumental de terceira geração tipo USS I e preencheram os critérios de inclusão do estudo. A avaliação da curva principal foi realizada pelo método de Cobb no pré-operatório, pós-operatório imediato e na última avaliação ambulatorial. As curvas foram classificadas

\section{ABSTRACT}

Objective: to assess and compare to the results reported in literature the outcomes of correction obtained in the immediate postoperative period and in the follow-up after arthrodesis consolidation in patients with adolescent idiopathic scoliosis who were submitted to Universal Spine System (USS I) hybrid instrumentation. Design of the study: series of cases. Methods: twenty patients with adolescent idiopathic scoliosis were submitted to instrumental fusion with third generation instrumentation (USS I). The radiographic evaluation was made through the Cobb's method in the pre-operative and immediate post-operative periods, and at the end of the treatment. The curves
\end{abstract}

\section{RESUMEN}

Objetivo: analizar y comparar con los hallazgos de la literatura los desenlaces de la corrección radiográfica en el plano coronal en adolescentes portadores de escoliosis idiopática torácica sometidos al tratamiento quirúrgico con instrumental de tercera generación tipo Universal Spine System (USS I). Diseño del estudio: estudio transversal. Serie de casos. Métodos: veinte adolescentes pacientes portadores de escoliosis idiopática del adolescente fueron sometidos a la artrodesis con instrumental de tercera generación tipo USS I y llenaron todos los criterios de inclusión del estudio. La evaluación de la curva principal fue realizada por el método de Cobb en el pre operatorio, postoperatorio inmediato y en la última

\footnotetext{
Grupo de Coluna do Departamento de Ortopedia e Traumatologia da Faculdade de Ciências Médicas da Santa Casa de São Paulo - FCMSCSP - São Paulo (SP), Brasil.

'Médico estagiário do Grupo de Coluna do Departamento de Ortopedia e Traumatologia da Faculdade de Ciências Médicas da Santa Casa de São Paulo FCMSCSP - São Paulo (SP), Brasil.

${ }^{2}$ Professora instrutora do Grupo da Coluna do Departamento de Ortopedia e Traumatologia da Faculdade de Ciências Médicas da Santa Casa de São Paulo FCMSCSP - São Paulo (SP), Brasil.

${ }^{3}$ Professor assistente e Chefe do Grupo da Coluna do Departamento de Ortopedia e Traumatologia da Faculdade de Ciências Médicas da Santa Casa de São Paulo - FCMSCSP - São Paulo (SP), Brasil.

${ }^{4}$ Consultor do Grupo da Coluna do Departamento de Ortopedia e Traumatologia da Faculdade de Ciências Médicas da Santa Casa de São Paulo - FCMSCSP São Paulo (SP), Brasil.
} 
segundo King e Lenke. Resultados: a média de seguimento foi de 20 meses. Este estudo mostrou $50 \pm 29 \%$ de correção da escoliose entre as radiografias pré e pós-operatórias imediatas. Entre o pós-operatório imediato e a última avaliação, foi demonstrada perda média de $11 \pm 16 \%$ da correção. Não foram observadas complicações, como infecção, soltura do implante, déficit neurológico ou pseudartrose. Conclusão: os resultados radiográficos encontrados foram similares aos da série de casos submetidos a outras estratégias de tratamento com uso de instrumentação de terceira geração. were classified based on King and Lenke's criteria. Results: the mean follow-up was 20 months. This study showed an immediate average

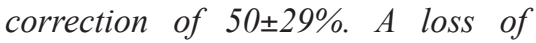
correction of $11 \pm 16 \%$ was observed during the follow-up. There were no complications such as infection, release of the implant, neurological deficit or pseudoarthrosis. Conclusion: the radiographic results showed that this instrumentation, regarding loss of correction in 20-month period, was similar to other methods using third generation instrumentation. evaluación ambulatoria. Las curvas fueron clasificadas según King y Lenke. Resultados: el promedio de seguimiento fue de 20 meses. Este es-

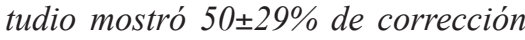
de la escoliosis entre las radiografias pre y postoperatorias inmediatas. Entre el postoperatorio inmediato y la última evaluación, fue demostrada una pérdida promedio de $11 \pm 16 \%$ de la correción. No fueron observadas complicaciones como infección, soltura del implante, déficit neurológico o pseudoartrosis. Conclusión: los resultados radiográficos encontrados fueron similares a la serie de casos sometidos a otras estrategias de tratamiento con uso de instrumentación de tercera generación.

DESCRIPTORES: Escoliosis/ cirugía; Escoliosis/ radiografía; Artrodesis/ métodos; Fusión vertebral/ métodos; Instrumentos quirúrgicos

\author{
DESCRITORES: Escoliose/ \\ cirurgia; Escoliose/ \\ radiografia; Artrodese/ \\ métodos; Fusão vertebral/ \\ métodos; Instrumentos \\ cirúrgicos
}

KEYWORDS: Scoliosis/surgery; Escoliosis/radiography; Arthrodesis/methods; Spinal fusion/methods; Surgical instruments

\section{INTRODUÇÃO}

A prevalência da escoliose é de 1 a $2 \%$ na população em geral, sendo que a escoliose idiopática do adolescente (EIA) é a sua apresentação mais frequente ${ }^{1}$.

Entre parentes de primeiro grau, a prevalência da EIA é de 5,21\%. O mais provável é que seja uma afecção com heterogeneidade genética envolvendo vários genes, alguns com herança mendeliana e outros obedecendo a uma herança multifatorial ${ }^{2}$.

As repercussões clínicas da EIA estão vinculadas à gravidade da deformidade. As deformidades que alcançam valor angular inferior ou igual a $20^{\circ}$ são consideradas leves e costumam acarretar somente queixas estéticas, não resultando em repercussões clínicas ou funcionais mais graves ${ }^{3}$.

Em casos com valores angulares superiores a $20^{\circ}$, podem ocorrer descompensação do tronco, repercussões respiratórias e progressão da deformidade mesmo após a maturação esquelética. Para o tratamento desses pacientes, o cirurgião ortopédico pode utilizar órteses ou a abordagem cirúrgica ${ }^{2-4}$.

As órteses são empregadas em pacientes com potencial de crescimento cuja escoliose atinja valores entre 20 e $40^{\circ}$. O tratamento cirúrgico costuma ser empregado em indivíduos com escolioses cujo valor angular seja superior a $40^{\circ}$. A história natural da escoliose idiopática do adolescente (EIA) e os benefícios a longo prazo da artrodese estão bem documentados na literatura ${ }^{5-8}$.
O princípio do tratamento cirúrgico da escoliose idiopática do adolescente é o mesmo da artrodese posterior, com a utilização do enxerto autólogo ${ }^{9,10}$.

O histórico do tratamento cirúrgico da escoliose com instrumental passou por evolução na linha do tempo com emprego dos instrumentais de Harrington et al., que submeteram pacientes às primeiras hastes para instrumentação ${ }^{11}$, e Luque, que introduziu o uso da instrumentação segmentar com fios sublaminares ${ }^{6}$.

Em 1984, o material de Cotrel-Dubousset (sistema de terceira geração) foi criado com o objetivo de atingir correção tridimensional da deformidade na coluna. Esse sistema tem a característica de manter o contorno no plano sagital e dispensar a necessidade do uso de imobilização no período pós-operatório. A característica principal desse implante é a estabilidade da montagem, o que contribui para uma maior taxa de consolidação da artrodese ${ }^{7}$.

$\mathrm{O}$ instrumental de Cotrel-Dubousset (CD) foi o primeiro sistema a empregar ganchos e parafusos e permitir fixação segmentar bilateral com distração e compressão seletiva em diferentes níveis ${ }^{12}$.

Em 1991, o Universal Spine System (USS) - Synthes ${ }^{\circledR}$ - foi desenvolvido. Esse sistema também usa ganchos e/ou parafusos e tem como princípio a correção da deformidade por meio da manobra de translação, na qual a coluna é trazida em direção à linha média do corpo ${ }^{13}$. 


\section{OBJETIVO}

Avaliar o desfecho radiográfico da correção coronal da curva principal de pacientes portadores de escoliose idiopática do adolescente submetidos ao tratamento cirúrgico com implante de terceira geração tipo USS I, com montagem híbrida, em nosso serviço.

\section{MÉTODOS}

Desenho do estudo: estudo transversal do tipo retrospectivo; série de casos.

Após aprovação pelo Comitê de Ética em Pesquisa com Seres Humanos do Serviço da Faculdade de Ciências Médicas da Santa Casa de São Paulo (protocolo 292/07), foram incluídos pacientes portadores de escoliose idiopática do adolescente, com curvas flexíveis torácicas à direita, não-estruturadas, de valor angular superior a $40^{\circ}$, com idade igual ou superior a 11 anos e seguimento mínimo de 1 ano, além de apresentar documentação radiográfica completa. Foram excluídos do estudo os pacientes submetidos à abordagem por via anterior ou outros tipos de curvas.

Foram avaliados 20 pacientes ( 3 do gênero masculino e 17 do feminino) portadores de escoliose idiopática do adolescente. A faixa etária média foi de 13 (11 a 16) anos de idade, no momento da abordagem cirúrgica.

$\mathrm{O}$ valor angular da curva principal foi mensurado em radiografia panorâmica, ortostática na incidência antereposterior segundo o método descrito por Cobb et al. ${ }^{14}$.

Todos os pacientes foram submetidos à correção cirúrgica da escoliose por via posterior, no Departamento de Ortopedia e Traumatologia da Santa Casa de Misericórdia de São Paulo, por membros do Grupo da Coluna entre 2002 e 2007.

O procedimento cirúrgico consistiu no posicionamento em: decúbito ventral; via de acesso posterior com exposição da área a ser instrumentada e submetida à artrodese; colocação de ganchos na coluna torácica e parafusos pediculares na toracolombar e lombar (montagem híbrida) com uso de material de terceira geração USS I Synthes ${ }^{\mathbb{R}}$; tornar cruenta a área óssea exposta e realização de artrodese pela técnica de Hibbs-Moe ${ }^{9}$ com colocação de enxerto autólogo $^{10}$ das cristas ilíacas posteriores. Após o término da correção da escoliose, foi empregado o teste do despertar de Stagnara ${ }^{15}$ no intraoperatório.

O padrão coronal das curvas foi classificado segundo King $^{16}$ (Tabela 1).
Todos os pacientes foram submetidos à avaliação após o seguimento mínimo de 12 meses, com variação de 12 a 32 meses (médio de 20 meses). Quando se observou integração do enxerto ósseo nos controles radiográficos oblíquos, a artrodese foi considerada consolidada ${ }^{17}$.

\section{RESULTADOS}

Os níveis de artrodese tiveram o limite proximal variando de T3 a T8 e distal de T12 a L4.

Não foram observadas complicações no período pósoperatório, como infecção, soltura do implante, déficit neurológico ou pseudoartrose.

Todas as curvas foram avaliadas segundo King, sendo agrupados como tipo II, III e IV (7, 18 e 2 pacientes, respectivamente).

Não houve descompensação no plano coronal.

A curva torácica apresentou valores angulares que variaram de 41 a $70^{\circ}$ (média de $58^{\circ} \pm 9^{\circ}$ ) na avaliação pré-operatória.

A correção obtida variou de 33 a $74 \%$ com média de $50 \pm 29 \%\left(50 \pm 8^{\circ}\right)$, e a perda da correção da curvatura variou de 1 a $28 \%$ (média de $11 \pm 16 \%$ ) (Tabela 2).

\section{DISCUSSÃO}

O principal objetivo do tratamento cirúrgico é impedir a progressão da deformidade mediante a artrodese. Esse objetivo poderia ser alcançado com eficiência mesmo no início do tratamento cirúrgico dessa deformidade, quando se empregava a artrodese seguida de aplicação de gesso ${ }^{18}$.

Com o desenvolvimento dos implantes metálicos, observou-se a possibilidade efetiva de correção da deformidade. O primeiro instrumental de ampla aceitação foi o sistema preconizado por Harrington (primeira geração). Esse método passou a ser difundido no final da década de $1950^{18}$.

Na década de 1970, a instrumentação com amarrilho sub-laminar de Luque (segunda geração) propiciou montagem segmentar mais rígida, passando a possibilitar a prevenção da deformidade com a síndrome de retificação da coluna que ocorre quando se emprega excessiva correção na coluna lombar ${ }^{8}$.

O método descrito por Cotrel e Dubousset (terceira geração), em $1984^{19}$, possibilita a correção da rotação vertebral. Este passou a ser o método de escolha no tratamento da escoliose com curva torácica simples, duplas curvas e curvas lombares, já que possibilita a correção da rotação

\section{TABELA 1 - Classificação das características da curvas da escoliose segundo King}

\begin{tabular}{ll}
\hline Tipo & Curva em S com ambas as curvas, torácica e lombar, cruzando a linha média \\
\hline 1 & Curva lombar maior que a torácica em radiografias em pé \\
2 & Curva em S com ambas as curvas, torácica e lombar, cruzando a linha média \\
3 & Curva torácica maior que a lombar \\
4 & Curva torácica longa, na qual L5 é centrada sobre o sacro e L4 inclina conforme a curva \\
5 & Curva torácica dupla com T1 inclinado em direção à convexidade da curva superior
\end{tabular}

Fonte: King HA, Moe JH, Bradford DS, Winter RB. The selection of fusion levels in thoracic idiophatic scoliosis. J Bone Joint Surg Am. 1983;65(9):1302-13(16). 


\begin{tabular}{|c|c|c|c|c|c|}
\hline Paciente & Inicial & $\begin{array}{l}\text { Pós-operatório } \\
\text { imediato }\end{array}$ & $\begin{array}{l}\text { Pós-operatório } \\
\text { tardio }\end{array}$ & $\begin{array}{c}\text { Correção } \\
\text { pós-operatória (\%) }\end{array}$ & Perda correção (\%) \\
\hline 1 & $65^{\circ}$ & $26^{\circ}$ & $39^{\circ}$ & 60 & 20 \\
\hline 2 & $50^{\circ}$ & $18^{\circ}$ & $32^{\circ}$ & 64 & 28 \\
\hline 3 & $61^{\circ}$ & $35^{\circ}$ & $36^{\circ}$ & 43 & 2 \\
\hline 4 & $70^{\circ}$ & $38^{\circ}$ & $32^{\circ}$ & 46 & 6 \\
\hline 5 & $49^{\circ}$ & $33^{\circ}$ & $36^{\circ}$ & 33 & 6 \\
\hline 6 & $65^{\circ}$ & $37^{\circ}$ & $38^{\circ}$ & 43 & 2 \\
\hline 7 & $50^{\circ}$ & $24^{\circ}$ & $26^{\circ}$ & 52 & 4 \\
\hline 8 & $58^{\circ}$ & $33^{\circ}$ & $35^{\circ}$ & 43 & 3 \\
\hline 9 & $51^{\circ}$ & $23^{\circ}$ & $28^{\circ}$ & 55 & 10 \\
\hline 10 & $55^{\circ}$ & $35^{\circ}$ & $40^{\circ}$ & 36 & 9 \\
\hline 11 & $68^{\circ}$ & $18^{\circ}$ & $40^{\circ}$ & 74 & 18 \\
\hline 12 & $41^{\circ}$ & $17^{\circ}$ & $24^{\circ}$ & 59 & 17 \\
\hline 13 & $55^{\circ}$ & $29^{\circ}$ & $36^{\circ}$ & 47 & 13 \\
\hline 14 & $54^{\circ}$ & $22^{\circ}$ & $32^{\circ}$ & 59 & 19 \\
\hline 15 & $68^{\circ}$ & $29^{\circ}$ & $39^{\circ}$ & 57 & 1 \\
\hline 16 & $69^{\circ}$ & $44^{\circ}$ & $45^{\circ}$ & 36 & 1 \\
\hline
\end{tabular}

Fonte: Serviço de Arquivo Médico da Santa Casa de São Paulo (SAME).

e a recuperação do balanço sagital enquanto propicia um sistema muito estável. Sua grande vantagem passa a ser a possibilidade de utilizar um sistema universal e bem adaptável $^{12}$.

Entretanto, como todo sistema, essa forma de tratamento também possui suas desvantagens. As principais são o maior risco de lesão neurológica, maior curva de aprendizagem e o custo elevado do instrumental. O risco de complicação neurológica com esse tipo de instrumental foi avaliado em aproximadamente 3\% dos casos segundo um levantamento do Scoliosis Research Society (SRS ) ${ }^{12}$.

A partir de sua introdução em 1991, o instrumental USS passou rapidamente a ser divulgado. Sua técnica baseavase na utilização de parafusos pediculares nos extremos das deformidades com ganchos na região intermediária. A correção da deformidade era realizada quando esses ganchos eram levados de encontro à barra, que estava estabilizada nos parafusos pediculares das extremidades ${ }^{13}$.

A partir disso, os parafusos pediculares e ganchos passaram a apresentar as mais diversas formas de utilização em sistemas somente com ganchos ou parafusos, ou mesmo em associações com ganchos e parafusos (híbridas).

Atualmente, o emprego das técnicas de instrumentação de implantes de terceira geração é de rotina na grande maioria dos centros que tratam essa afecção ${ }^{20}$.

Verificou-se, na literatura, ampla variação nos valores de correção e perda da correção coronal da EIA; entretanto, vale ressaltar que existe diferença na estratégia de instrumentação dos implantes de terceira geração entre os diferentes autores. Com uso de ganchos, a correção varia de 33 a $51 \%$ e a perda pós-operatória varia de 8 a 13\% ${ }^{19-22}$; com parafusos pediculares, a correção varia de 44 a $76 \%$ e a perda de correção varia de 5 a $9 \% \%^{23-24}$; e com sistema híbrido, a correção média é de $56 \%$ e a perda de correção média é de $10 \%{ }^{20}$. Para comparar, observou-se $50 \%$ de correção da escoliose entre as radiografias pré e pósoperatórias imediatas. Entre o pós-operatório imediato e a última avaliação, foi demonstrada perda média de $11 \%$ da correção.

A vantagem da instrumentação híbrida, em comparação a outros métodos de instrumentação, é a possibilidade de correção satisfatória da deformidade sem a necessidade de utilização de parafusos pediculares na coluna torácica, na qual há maior risco inerente de lesão iatrogênica da medula espinal ${ }^{23}$.

Apesar de a avaliação radiográfica dos valores angulares de correção e de a perda da correção nos diferentes tipos de montagem serem discutidas na literatura, deve-se enfatizar a necessidade de ensaios clínicos futuros randomizados com parâmetros clínicos de avaliação centrados nos pacientes para auxiliar a definir o melhor tratamento para esses pacientes. Contudo, com base nesta experiência e nos achados da literatura, esta técnica revelou-se segura e satisfatória para a correção coronal radiográfica da deformidade nos portadores de EIA.

\section{CONCLUSÃO}

Os resultados radiográficos de correção e perda da correção no tratamento cirúrgico de escoliose idiopática do adolescente com instrumentação híbrida foram similares aos da série de casos submetidos a outras estratégias de tratamento com instrumentação de terceira geração. 


\section{REFERÊNCIAS}

1. Lonstein JE, Bjourklund S, Wanninger MH, Nelson RP. Voluntary school screening for scoliosis in Minnesota. $\mathrm{J}$ Bone Joint Surg Am. 1982;64(4): 481-8.

2. Wajchenberg M, Puertas EB, Zatz M. Estudo da prevalência da escoliose idiopática do adolescente em pacientes brasileiros. Coluna/Columna. 2005;4(3):113-68.

3. Weinstein SL, Ponseti IV. Curve progression in idiopathic scoliosis. J Bone Joint Surg Am. 1984;65(4): 447-55.

4. Ascani E, Bartolozzi P, Logroscino CA, Marchetti PG, Ponte A, Savini $\mathrm{R}$, et al. Natural history of untreated idiopathic scoliosis after skeletal maturity. Spine. 1986;11(8):784-9.

5. Carr WA, Moe JH, Winter RB, Lonstein JE. Treatment of idiopathic scoliosis in the Milwaukee brace. J Bone Joint Surg Am. 1980;62: 599-612.

6. Nachemson AL, Peterson LE. Effectiveness of treatment with a brace in girls who have adolescent idiopathic scoliosis: a prospective, controlled study based on data from Brace Study of the Scoliosis Research Society. J Bone Joint Surg Am. 1995;77(6): 815-22.

7. Lonstein LE, Carlson JM. The prediction of curve progression in untreated scoliosis during growth. J Bone Joint Surg Am. 1984;66(7): 1061-71.

8. Mielke CH, Lonstein JE, Denis F, Vandenbrink K, Winter PB. Surgical treatment of adolescent idiopathic scoliosis. J Bone Joint Surg. 1989;71(8):1170-7.

9. Hibbs RA. A report of fifty-nine cases of scoliosis treated by the fusion operation. J Bone Joint Surg Am. 1924;6:3-34.

10.Moe JH. A critical analysis of methods of fusion for scoliosis: an evolution in two hundred and sixty-six patients. J Bone Joint Surg Am. 1958;40:529697.
11. Harrington PR. Treatment of scoliosis: correction and internal fixation by spine instrumentation. J Bone Joint Surg Am. 1962;44-A:591-634.

12.Kostuik JP. Operative treatment of idiopathic scoliosis. J Bone and Joint Surg Am. 1990;72(7):1108-13.

13. Mohan AL, Das K. History of surgery for correction of spinal deformity. Neurosurg Focus. 2003;14(1):e1.

14.Cobb JR. Outline for the Study of Scoliosis. In: Instructional Course Lectures, The American Academy of Orthopaedics Surgeons 1984;5: 261-75.

15.Hall JE, Levine CR, Sudhir KG. Intraoperative awakening to monitor spinal cord function during Harrington instrumentation and spine fusion. Description of procedure and report of three cases. J Bone Joint Surg Am. 1968;60(4):533-6.

16.King HA, Moe JH, Bradford DS, Winter RB. The selection of fusion levels in thoracic idiophatic scoliosis $\mathrm{J}$ Bone Joint Surg Am. 1983;65(9): 1302-13.

17.Dawson EG, Clader TJ, Bassett LW. A comparison of different methods used to diagnose pseudoarthrosis following posterior spinal fusion scoliosis. J Bone Joint Surg Am. 1985;67(8): 1153-9.

18.Dickson JH, Harrington PR. The Evolution of the Harrington Instrumentation Technique in Scoliosis. J Bone Joint Surg Am. 1973;55(5):993-1002.

19.Kim YJ, Lenke LG, Cho SK, Bridwell KH, Sides B, Blanke K. Comparative analysis of pedicle screw versus hook instrumentation in posterior fusion of adolescent idiophatic scoliosis. Spine. 2004;29(18):2040-8.

20.Lenke LG, Bridwell KH, Baldus C, Blanke K, Schoenecker PL. CotrelDubousset instrumentation for adolescent idiopathic scoliosis. J Bone Joint Surg Am. 1992;74(7):1056-67.
21.Liljenqvist U, Lepsien U, Hackenberg L. Comparative analysis of pedicle screw and hook instrumentation in posterior correction and fusion of idiopathic thoracic scoliosis. Eur Spine J. 2002;11(4):336-43.

22.Dobbs MB, Lenke LG, Kim YJ, Kamath G, Peelle MW, Bridwell KH. Selective posterior thoracic fusion for adolescent idiopathic scoliosis. Spine. 2006;31(20):2400-4.

23. Vaccaro AR, Rizzolo SJ, Balderston RA, Allardyce TJ, Garfin SR, Dolinskas C. Placement of pedicle screws in the thoracic spine. Part II: an anatomic and radiographic assessment. J Bone Joint Surg Am. 1995;77(8):1200-6.

24.Avanzi O, Chih LY, Meves R, Caffaro MFS, Rangel TAM. Avaliação estético-funcional dos portadoresde escoliose idiopática do adolescente à instrumentação com implante metálico de Cotrel-Dubousset . Coluna/ Columna. 2006;5(3):148-51.

\section{Correspondência:}

\section{Robert Meves}

Rua Dr. Cesário Mota Júnior, 112 Vila Buarque

CEP $01277-900$ - São Paulo (SP), Brasil

Fone: (11) 2176-7000, ramal 1565

Email: robertmeves@santacasasp.org.br, coluna@santacasasp.org.br 